\title{
An ONIOM study of the Bergman reaction: a computationally efficient and accurate method for modeling the enediyne anticancer antibiotics
}

\author{
Steven Feldgus *, George C. Shields \\ Department of Chemistry, Hamilton College, 198 College Hill Road, Clinton, NY 13323, USA
}

Received 26 June 2001; in final form 8 August 2001

\begin{abstract}
The Bergman cyclization of large polycyclic enediyne systems that mimic the cores of the enediyne anticancer antibiotics was studied using the ONIOM hybrid method. Tests on small enediynes show that ONIOM can accurately match experimental data. The effect of the triggering reaction in the natural products is investigated, and we support the argument that it is strain effects that lower the cyclization barrier. The barrier for the triggered molecule is very low, leading to a reasonable half-life at biological temperatures. No evidence is found that would suggest a concerted cyclization/H-atom abstraction mechanism is necessary for DNA cleavage. (c) 2001 Elsevier Science B.V. All rights reserved.
\end{abstract}

\section{Introduction}

The enediyne family of natural products is a group of DNA cleaving molecules that exhibits tremendous potential as a source of anticancer drugs [1]. Members of this family include the esperamicins (Fig. 1), calicheamicins, dynemycins, and the neocarzinostatin chromophore. In the commonly accepted mechanism for Esperamicin $\mathrm{A}_{1}$, nucleophilic attack on the trisulfide 'trigger' generates a thiol, which undergoes intramolecular addition to the 10 -membered ring containing the enediyne. This induces a Bergman cyclization [2] of the enediyne, generating a reactive diradical spe-

\footnotetext{
${ }^{*}$ Corresponding author. Fax: +1-315-859-4744.

E-mail address: sfeldgus@hamilton.edu (S. Feldgus).
}

cies that abstracts hydrogen atoms from the DNA backbone, leading to DNA cleavage.

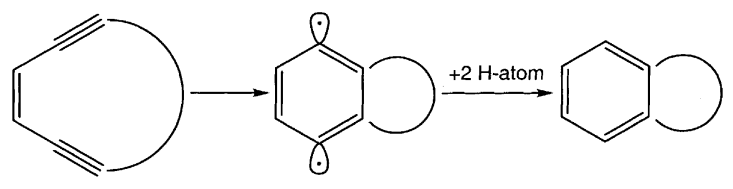

Unfortunately, the enediyne natural products will cleave the DNA of healthy cells as well [3], limiting their usefulness as cancer drugs. It may be possible to increase the selectivity of enediynes by harnessing the $\mathrm{pH}$ difference between healthy and cancerous cells. Cancer cells are generally more acidic [4], and Hoffner et al. found that enediynes with one ene carbon replaced by nitrogen cyclize only when protonated. This has sparked attempts 


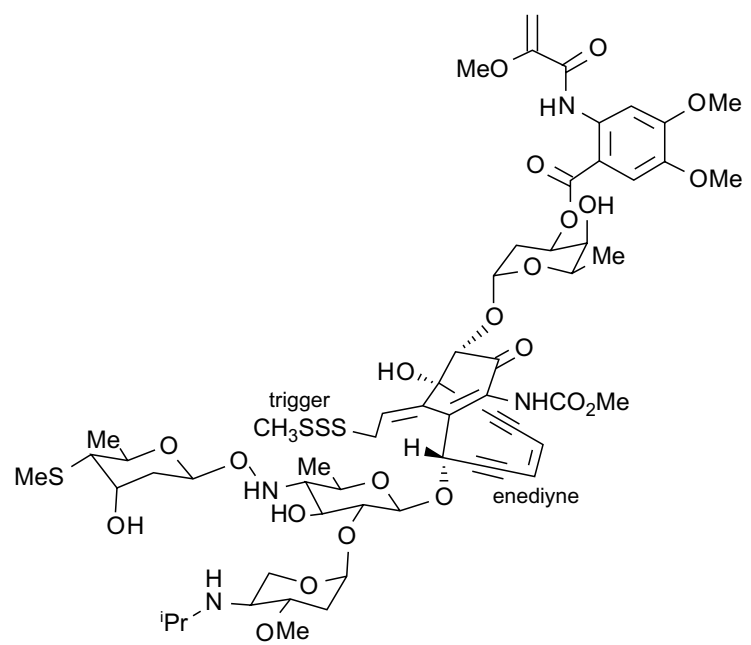

Fig. 1. Esperamicin $A_{1}$, a member of the enediyne family of natural anticancer antibiotics, with the enediyne and trigger sections labeled.

to computationally design novel $\mathrm{pH}$-dependent enediyne drugs $[5,6]$. The criteria for determining the suitability of new molecules are based on the commonly accepted mechanism mentioned above. However, there are at least two other possibilities. The first, proposed by Lindh and Persson [7], is hydrogen atom abstraction from DNA coincident with enediyne cyclization. This is supported by the enhanced stability of diradicals relative to monoradicals [8], which arises from singlet coupling between the two unpaired electrons. If the gap between the less-reactive singlet and more-reactive triplet state is too large, the diradical would be too stable to abstract hydrogen rapidly via the stepwise mechanism. The second possibility is based on recent evidence that diradicals generated in biological conditions react with molecular oxygen to form quinones faster than with hydrogen atom donors to form stable arenes [9]. This raises the possibility that a quinone is responsible for enediyne-induced DNA cleavage, instead of the diradical itself. Understanding the actual mechanism of the natural products is necessary to guide efforts to design new drugs.

The discovery of the enediyne natural products initiated a flurry of computational activity aimed at understanding the details of the Bergman cyclization. The earliest work used molecular me- chanics to study large fragments of the natural products [10,11], while more recent studies have used high level electronic structure methods to accurately model the cyclization of small enediynes [7,12-18]. The Bergman cyclization is a difficult reaction to model computationally because of the multiconfigurational nature of the diradical. Molecular mechanics is completely unsuitable for obtaining accurate energies of the natural products, while methods such as $\operatorname{CCSD}(\mathrm{T})$ are far too expensive to be used on anything but small model compounds. DFT is a promising high-accuracy, low-cost alternative, and has been used effectively in the past few years to model the cyclizations of larger enediynes that are more similar in structure to the natural products [5,17-19]. One DFT study of the triggering reaction in the calicheamicin $\gamma_{1}^{\mathrm{I}}$ core has been published, but it does not model transition states or the biradicals [20].

In this study, we apply the ONIOM hybrid method [21] to triggered and untriggered polycyclic systems similar to those found at the core of natural enediynes. The ONIOM method allows us to use a combination of expensive and inexpensive theories to make high accuracy computations possible on large molecules. We will show that ONIOM is appropriate for modeling these types of systems, and make some preliminary insights into the effect of the triggering reaction and the energetics of the Bergman reaction in the natural products. This is a first step towards gaining a more detailed understanding of the mechanism of DNA cleavage by the enediyne natural products.

\section{Computational methods}

Geometries were obtained with the B3LYP density functional method and the 6-31G(d,p) basis set. This method provides excellent geometries for enediynes and diradicals that do not change significantly upon expansion of the basis set [5]. Hybrid density functional methods, such as B3LYP, had initially been declared inappropriate for modeling diradical species [16-18], but that conclusion was based on the performance of restricted B3LYP, and Gräfenstein et al. [15] recently demonstrated that unrestricted B3LYP 
performs better than pure density functionals such as BLYP. Energies were obtained via the ONIOM hybrid QM/MM method as implemented in Gaussian 98 [22]. The ONIOM energy for a twolayer system is calculated by

$$
\begin{aligned}
E(\text { Real }: \text { High })= & E(\text { Core }: \text { High })+[E(\text { Real }: \text { Low }) \\
& -E(\text { Core }: \text { Low })]
\end{aligned}
$$

where Real and Core indicate the molecular layers, and High and Low indicate the level of theory. The six-carbon enediyne moiety was chosen as the ONIOM core, and the High level of theory was the coupled-cluster theory with Brueckner orbitals $(\mathrm{BD}(\mathrm{T}))$ and the cc-pVDZ basis set. Coupledcluster methods have performed extremely well in previous enediyne studies, closely matching experimental energetic [13] and geometric [23] data, and Cramer found that $\mathrm{BD}(\mathrm{T})$ was more stable than $\operatorname{CCSD}(\mathrm{T})$ for low symmetry biradicals [16]. The Low level method was B3LYP/cc-pVTZ. In the tables, $\Delta E$ values refer to the calculated electronic energy differences at $0 \mathrm{~K}$ between the uncyclized reactant and the diradical product $\left(\Delta E_{r x n}\right)$, or between the reactant and the cyclization transition state $\left(\Delta E^{\ddagger}\right)$. Enthalpies and free energies were calculated by taking the thermochemistry output from unscaled Gaussian frequency calculations at the temperature of interest and adding them to the electronic energies. All structures had the correct number of negative vibrational fre- quencies: zero for minima, one for transition states.

\section{Results and discussion}

\subsection{Computational benchmarks}

We conducted a set of computational benchmark calculations on small enediyne molecules for which experimental data is available: cis-hex-3ene-1,5-diyne (1), o-diethynylbenzene (2) and cyclodec-3-ene-1,5-diyne (3).
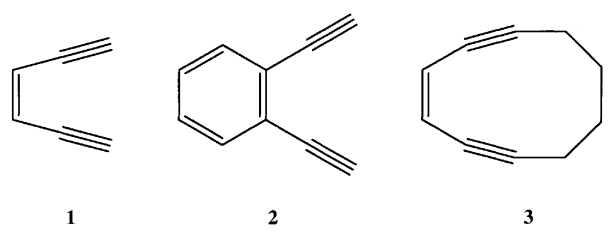

Results for these molecules are collected in Table 1. In all cases, B3LYP/6-31G(d,p) underestimates the enthalpy of reaction but overestimates the enthalpic barrier. B3LYP/cc-pVTZ single point energies overestimate both $\Delta H_{r x n}$ and $\Delta H^{\ddagger}$. $\mathrm{BD}(\mathrm{T})$ single points are more accurate than either B3LYP calculation, but at a tremendous computational cost. The ONIOM calculations are also more accurate than the B3LYP calculations, more

Table 1

\begin{tabular}{|c|c|c|c|c|c|c|}
\hline & \multicolumn{2}{|l|}{1} & \multicolumn{2}{|l|}{2} & \multicolumn{2}{|l|}{3} \\
\hline & $\Delta H_{r x n}$ & $\Delta H^{\ddagger}(470 \mathrm{~K})$ & $\Delta H_{r x n}$ & $\Delta H^{\ddagger}(486 \mathrm{~K})$ & $\Delta H_{r x n}$ & $\Delta H^{\ddagger}(298 \mathrm{~K})$ \\
\hline B3LYP/6-31G(d,p) & 3.0 & 29.4 & 12.0 & 29.5 & 3.3 & 26.0 \\
\hline B3LYP/cc-pVTZ//6-31G(d,p) & 10.0 & 34.2 & 18.6 & 33.0 & 9.6 & 29.7 \\
\hline $\mathrm{BD}(\mathrm{T}) / \mathrm{cc}-\mathrm{pVDZ} / / 6-31 \mathrm{G}(\mathrm{d}, \mathrm{p})^{\mathrm{a}}$ & 5.3 & 27.6 & 15.4 & $-{ }^{\mathrm{f}}$ & $-{ }^{f}$ & 21.6 \\
\hline $\mathrm{ONIOM}(\mathrm{BD}(\mathrm{T}): \mathrm{B} 3 \mathrm{LYP})^{\mathrm{b}}$ & - & - & 14.7 & 27.0 & 6.2 & 22.9 \\
\hline Experimental & $8.5 \pm 1.0^{\mathrm{c}}$ & $28.2 \pm 0.5^{\mathrm{c}}$ & $17.8 \pm 1.2^{\mathrm{d}}$ & $25.2 \pm 0.8^{\mathrm{d}}$ & - & $23.2^{\mathrm{e}}$ \\
\hline
\end{tabular}

Enthalpies $(\mathrm{kcal} / \mathrm{mol})$ of activation and reaction for three benchmark molecules

All values in $\mathrm{kcal} / \mathrm{mol}$. All geometries and frequencies obtained at the B3LYP/6-31G(d,p) level of theory.

${ }^{\mathrm{a}}$ Indicates single point energy at indicated level of theory on B3LYP/6-31G(d,p) geometry.

${ }^{\mathrm{b}}$ Indicates ONIOM calculation with BD(T)/cc-pVDZ at High level of theory and B3LYP/cc-pVTZ as Low level of theory.

${ }^{\mathrm{c}}$ Ref. [33].

${ }^{\mathrm{d}}$ Ref. [34].

${ }^{\mathrm{e}}$ Ref. [10], using relationship between $E_{\mathrm{a}}$ and $H$ for unimolecular reaction $\left(\Delta H^{\ddagger}=E_{\mathrm{a}}-R T\right)$ [35].

${ }^{\mathrm{f}}$ Not calculated due to computational time constraints. 
accurate than $\mathrm{BD}(\mathrm{T})$ for $\Delta H^{\ddagger}$ of $\mathbf{3}$, and are $10-25$ times quicker than the full $\mathrm{BD}(\mathrm{T})$ calculations.

\subsection{Geometry of model systems}

The active region of the calicheamicin and esperamicin families are nearly identical: the methyl(1,8,9-trihydroxy-13-[7,3,1]trideca-4,9-diene-2, 6-diyne-10-yl)carbonate (MTC) 'warhead' (in calicheamicin, $\mathrm{O}^{*}$ is a hydrogen).

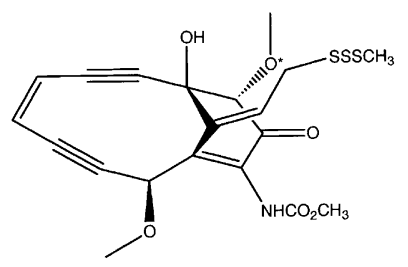

MTC

We constructed two model systems based on this structure (Fig. 2). One is an untriggered structure without the $\mathrm{CH}_{2} \mathrm{SSSCH}_{3}$ moiety entirely, MTC-UT, and one is a triggered structure, MTCTR, where the thiol has undergone intramolecular addition. In both of these, hydrogens have replaced the oxygens that connect MTC to the saccharide chains in the natural products. We performed full B3LYP geometry optimizations on MTC, MTC-UT, and MTC-TR. We also performed an ONIOM geometry optimization on the entire Esperamicin $\mathrm{A}_{1}$ molecule, using the link oxygens in MTC as the boundary between the high and low layers, and treating the low layer with the semiempirical method AM1. The structure of Esperamicin $A_{1}$ bound to DNA in solution has been published as a set of four NMR-refined molecular dynamics geometries [24]. We averaged the four solution geometries and compared the bond lengths and angles of the carbon atoms on the 10-membered ring, plus the $\mathrm{C}_{1}-\mathrm{C}_{6}$ and $\mathrm{C}_{2}-\mathrm{C}_{5}$ distances, to our optimized geometries. The mean absolute and root-mean-square deviations are given in Table 2, and show excellent agreement between our computational structures and the empirically derived ones, independent of system size. The solution structure of calicheamicin $\gamma_{1}^{\mathrm{I}}$ bound to DNA has also been published as a set of six geometries [25]. However, the geometry of the 10 -membered ring within calicheamicin is considerably different than in esperamicin, and the deviations between our structures and calicheamicin are approximately twice as large. This is presumably due to the structural effect of DNA on the MTC region of these molecules, with calicheami$\operatorname{cin} \gamma_{1}^{\mathrm{I}}$ being affected more than Esperamicin $\mathrm{A}_{1}$. The influence of DNA and solvent molecules on the geometry of these molecules is currently under investigation.

The transition state for the Bergman cyclization and the diradical product were calculated for MTC-UT and MTC-TR. The optimized geometries for MTC-TR are shown in Figs. 3 and 4. The

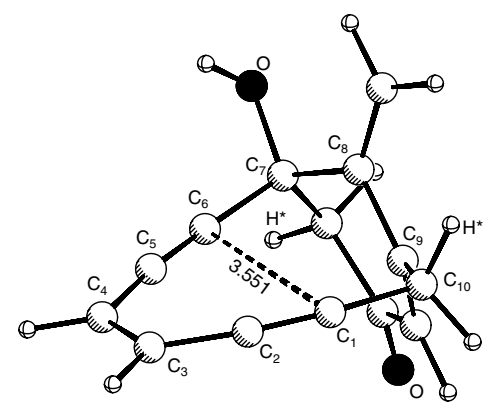

MTC-UT

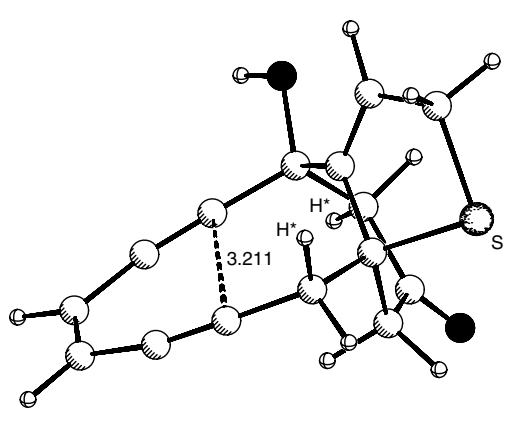

MTC-TR

Fig. 2. B3LYP/6-31G(d,p) optimized structures of untriggered (left) and triggered (right) enediynes. The labels on the untriggered structure indicate the numbering scheme used in this paper. Hydrogens marked with an asterisk are the link points to the rest of the molecule. 
Table 2

Deviations of 10-membered ring geometries of B3LYP and ONIOM optimized structures from the average of four experimental solution structures of Esperamicin $\mathrm{A}_{1}$ bound to DNA

\begin{tabular}{llllll}
\hline Geometry & Distances $(\AA)$ & & Angles $(\mathrm{deg})$ \\
\cline { 2 - 3 } \cline { 5 - 5 } & MAD & RMSD & MAD & RMSD \\
\hline B3LYP/MTC & 0.020 & 0.024 & 1.847 & 2.462 \\
B3LYP/MTC-UT & 0.021 & 0.028 & 1.918 & 2.315 \\
ONIOM (B3LYP/6-31G(d,p):AM1) & 0.022 & 0.025 & 2.203 & 2.712 \\
\hline
\end{tabular}

Solution structures from [24].

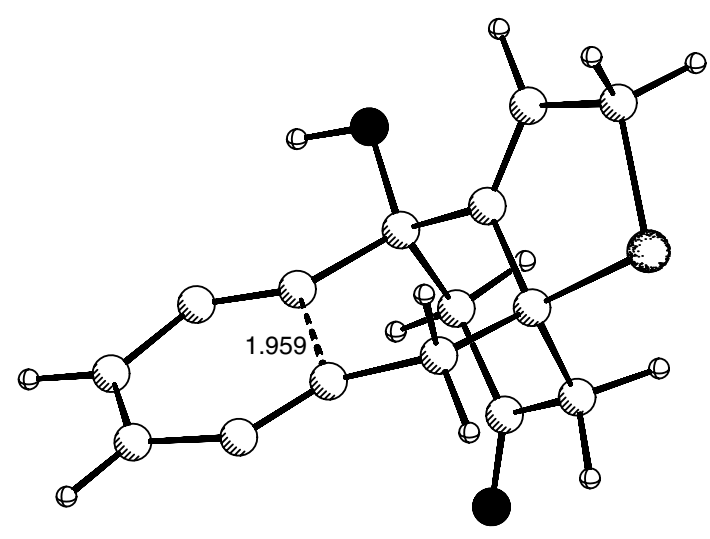

Fig. 3. UB3LYP/6-31G(d,p) optimized structure of the transition state for cyclization of MTC-TR.

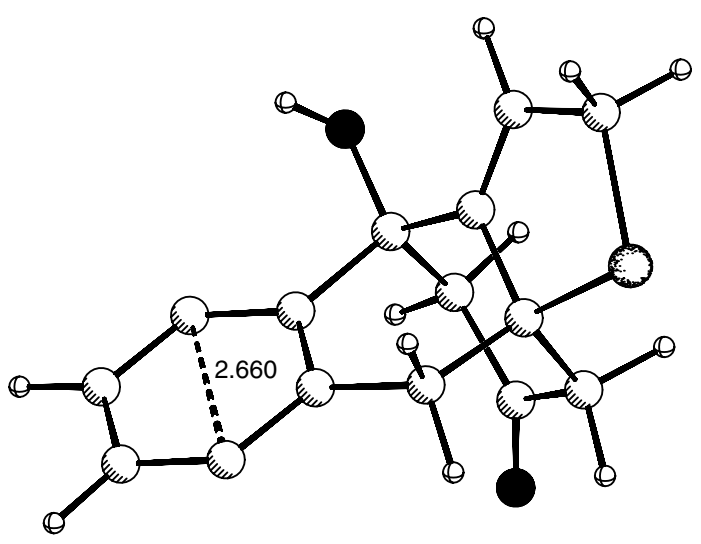

Fig. 4. UB3LYP/6-31G(d,p) optimized geometry of the unrestricted singlet geometry of the MTC-TR diradical.

transition state for MTC-TR has a very small amount of diradical character $\left(\left\langle S^{* *} 2\right\rangle=0.07\right)$, which is in agreement with other studies on the Bergman cyclization that have found early transi- tion states with little or no diradical character $[13,16]$. The Hammond postulate predicts a later transition state with more diradical character for the highly endothermic (see below) cyclization of MTC-UT, and we do see considerably more spin contamination in that transition state $\left(\left\langle S^{* *} 2\right\rangle=\right.$ $0.29)$. The spin contamination of both singlet diradicals is about the same $\left(\left\langle S^{* *} 2\right\rangle=0.97\right)$, which is similar to the spin contamination values found for unrestricted B3LYP calculations on other small enediynes [15].

\subsection{Energetics of model systems}

The calculated energies of cyclization for our model systems are given in Table 3. The influence of triggering on lowering the barrier to cyclization is dramatic. Two different theories have been proposed to explain this effect. The first says that the barrier to cyclization drops along with the distance between the terminal acetylinic carbons $\left(\mathrm{C}_{1}-\mathrm{C}_{6}\right)$, and that below a threshold distance cyclization is spontaneous $[10,18]$. The second theory claims that triggering leads to reduced strain and increased flexibility in the enediyne ring, which results in a lower barrier [11,20,26]. Our $\mathrm{BD}(\mathrm{T})$ results on the six-carbon enediyne cores clearly show that there is very little change in the barrier to cyclization after triggering, even though the $\mathrm{C}_{1}-\mathrm{C}_{6}$ distance drops from 3.55 to $3.21 \AA$. Furthermore, the $\mathrm{C}_{1}-\mathrm{C}_{6}$ distance in $\mathbf{3}$ is $3.39 \AA$, yet the $\mathrm{BD}(\mathrm{T})$ barrier to cyclization of the six-carbon core is $19.98 \mathrm{kcal} / \mathrm{mol}$, which is $0.4 \mathrm{kcal} / \mathrm{mol}$ less than MTC-TR. This suggests that the presence of the double bond at $\mathrm{C}_{9}$ is responsible for the large barrier in MTC-UT, and strongly supports the notion that the distance between the two carbons is not an accurate predictor of the cyclization 
Table 3

Energetics $(\mathrm{kcal} / \mathrm{mol})$ of cyclization of MTC-UT and MTC-TR molecules

\begin{tabular}{|c|c|c|c|c|c|c|c|c|}
\hline \multirow[t]{2}{*}{ Method } & \multicolumn{4}{|c|}{ MTC-UT (untriggered) } & \multicolumn{4}{|c|}{ MTC-TR (triggered) } \\
\hline & $\Delta E_{r x n}$ & $\Delta H_{r x n}$ & $\Delta E^{\ddagger}$ & $\Delta H^{\ddagger}$ & $\Delta E_{r x n}$ & $\Delta H_{r x n}$ & $\Delta E^{\ddagger}$ & $\Delta H^{\ddagger}$ \\
\hline B3LYP/6-31G(d,p) & 25.35 & 24.65 & 45.82 & 43.54 & -3.04 & -2.93 & 21.78 & 20.12 \\
\hline B3LYP/cc-pVTZ & 31.29 & 30.59 & 49.66 & 47.32 & 3.27 & 3.38 & 25.59 & 23.93 \\
\hline $\begin{array}{l}\text { B3LYP/cc-pVTZ on } \\
\text { ONIOM core }\end{array}$ & 4.45 & 3.74 & 30.50 & 28.16 & 2.19 & 2.30 & 28.23 & 26.57 \\
\hline $\mathrm{BD}(\mathrm{T})$ on $\mathrm{ONIOM}$ core & 0.70 & -0.01 & 23.16 & 20.82 & -0.87 & -0.77 & 22.04 & 20.38 \\
\hline ONIOM $(\mathrm{BD}(\mathrm{T}): \mathrm{B} 3 \mathrm{LYP})^{\mathrm{b}}$ & 27.54 & 26.84 & 42.32 & 39.97 & 0.21 & 0.31 & 19.40 & 17.74 \\
\hline
\end{tabular}

${ }^{\mathrm{a}} \mathrm{B} 3 \mathrm{LYP} / \mathrm{cc}-\mathrm{pVTZ}$ single point on entire molecule.

${ }^{\mathrm{b}}$ Indicates BD(T)/cc-pVDZ as High level of theory and B3LYP/cc-pVTZ as Low level of theory. The ONIOM value is the B3LYP/ccpVTZ ONIOM core energy minus the B3LYP/cc-pVTZ single point energy, plus BD(T) on the core. Frequencies taken, unscaled, from the B3LYP/6-31G(d,p) geometries for estimation of thermochemical parameters.

barrier, at least in cyclic enediynes. Recent results suggest it is not an accurate predictor in acyclic enediynes either [27].

The trends seen in Table 3 are similar to those from Table 1. Relative to the ONIOM calculations, B3LYP/6-31G(d,p) underestimates the endothermicity and overestimates the barrier, while B3LYP/cc-pVTZ overestimates both. This is not inherent to the ONIOM method, but is sensitive to the relative magnitudes of $\mathrm{BD}(\mathrm{T})$ and $\mathrm{B} 3 \mathrm{LYP}$ energies. The calculated ONIOM free energy of activation $\left(\Delta G^{\ddagger}=18.67 \mathrm{kcal} / \mathrm{mol}\right.$ at $\left.262 \mathrm{~K}\right)$ is close to the experimental free energy of activation of a 10 -membered dihydrothiophene $\operatorname{ring}\left(\Delta G^{\ddagger}=19.4\right.$ $\mathrm{kcal} / \mathrm{mol}$ at $262 \mathrm{~K}$ ) that has a similar structure to MTC-TR [28]. The calculated free energy of activation for MTC-TR at body temperature $(310 \mathrm{~K})$ is $18.84 \mathrm{kcal} / \mathrm{mol}$, which is equivalent to a half-life of approximately $2 \mathrm{~s}$. This is rapid enough to account for the biological activity of the triggered molecules without having to invoke a concerted cyclization/abstraction mechanism.

The reason for the lower barrier in MTC-TR relative to 3 is unclear at this point. One possibility is the presence of the hydroxyl group at $\mathrm{C}_{7}$. Electron withdrawing groups near the alkyne carbons accelerate the cyclization of enediynes, potentially through decreased in-plane $\pi$-electron repulsion [29,30]. Addition of a hydroxy group to the $C_{7}$ position of 3 speeds the reaction up significantly [31]. Since we eliminated two oxygens from esperamicin in generating our model structure, one in the $\mathrm{C}_{10}$ position, the real barriers for the natural product might be even lower.
As mentioned in Section 1, the singlet-triplet gaps of the natural product diradicals can be a predictor of their reactivity. Both $\mathrm{BD}(\mathrm{T})$ and $\mathrm{DFT}$ methods have matched the experimental singlettriplet gap of 1 (singlet $3.8 \mathrm{kcal} / \mathrm{mol}$ below triplet [32]) to within $\sim 1 \mathrm{kcal} / \mathrm{mol}[5,16]$. The calculated singlet-triplet gaps for our model systems are small, ranging from $1.36 \mathrm{kcal} / \mathrm{mol}$ for the $\mathrm{ON}$ $\operatorname{IOM}(\mathrm{BD}(\mathrm{T})$ :B3LYP) level to 2.37 for B3LYP/ccpVTZ on the ONIOM core. This small energetic difference between states indicates that the natural products should not a priori be expected to be less reactive with respect to hydrogen atom abstraction than small symmetric diradicals such as $p$-benzyne. Other computational studies have found similarly small singlet-triplet gaps in large asymmetric enediynes [5].

\section{Conclusion}

We have shown that density functional theory and Bruecker coupled-cluster theory can be combined within the ONIOM hybrid method to investigate the behavior of natural-product-like enediynes. The ONIOM results are comparable to $\mathrm{BD}(\mathrm{T}) / \mathrm{cc}-\mathrm{pVDZ}$ results for small enediynes while being significantly faster. B3LYP/6-31G(d) provides excellent geometries for larger enediynes, and the ONIOM calculations indicate that the cyclization of the natural products should be approximately thermoneutral. The effect of the triggering reaction on lowering the cyclization barrier comes from the change in the strain energy of the ring, 
not simply from the reduced distance between $\mathrm{C}_{1}$ and $\mathrm{C}_{6}$. Our results are consistent with a mechanism that involves stepwise cyclization followed by $\mathrm{H}$-atom abstraction, but cannot distinguish between that and possible quinone formation. The performance of ONIOM in this work is encouraging for our continuing studies of the enediyne natural products.

\section{Acknowledgements}

We acknowledge NIH, the Camille and Henry Dreyfus Scholar/Fellow Program for Undergraduate Institutions, and Hamilton College for support of this work. Computer time was obtained from the National Computational Science Alliance under CHE010017N and utilized the Origin 2000 supercomputers.

\section{References}

[1] K.C. Nicolaou, W.-M. Dai, Angew. Chem. Int. Ed. Engl. 30 (1991) 1387.

[2] R.G. Bergman, Acc. Chem. Res. 6 (1973) 25.

[3] A.L. Smith, K.C. Nicolaou, J. Med. Chem. 39 (1996) 2103.

[4] J.L. Wike-Hooley, J. Haveman, H.S. Reinhold, Radiotherapy Oncol. 2 (1984) 343.

[5] E. Kraka, D. Cremer, J. Am. Chem. Soc. 122 (2000) 8245.

[6] E. Kraka, D. Cremer, J. Comput. Chem. 22 (2001) 216.

[7] R. Lindh, B.J. Persson, J. Am. Chem. Soc. 116 (1994) 4963.

[8] M.J. Schottelius, P. Chen, J. Am. Chem. Soc. 118 (1996) 4896.

[9] L.H. Jones, C.W. Harwig, J.P. Wentworth, A. Simeonov, A.D. Wentworth, S. Py, J.A. Ashley, R.A. Lerner, K.D. Janda, J. Am. Chem. Soc. 123 (2001) 3607.

[10] K.C. Nicolaou, G. Zuccarello, Y. Ogawa, E.J. Schweiger, T. Kumazawa, J. Am. Chem. Soc. 110 (1988) 4866.

[11] P. Magnus, S. Fortt, T. Pitterna, J.P. Snyder, J. Am. Chem. Soc. 112 (1990) 4986.
[12] N. Koga, K. Morokuma, J. Am. Chem. Soc. 113 (1991) 1907.

[13] E. Kraka, D. Cremer, J. Am. Chem. Soc. 116 (1994) 4929.

[14] R. Lindh, T.J. Lee, A. Bernhardsson, B.J. Persson, G. Karlström, J. Am. Chem. Soc. 117 (1995) 7186.

[15] J. Gräfenstein, A.M. Hjerpe, E. Kraka, D. Cremer, J. Phys. Chem. A 104 (2000) 1748.

[16] C.J. Cramer, J. Am. Chem. Soc. 120 (1998) 6261.

[17] W.-C. Chen, N.-Y. Chang, C.-H. Yu, J. Phys. Chem. A 102 (1998) 2584

[18] P.R. Schreiner, J. Am. Chem. Soc. 120 (1998) 4184.

[19] G.B. Jones, P.M. Warner, J. Am. Chem. Soc. 123 (2001) 2134.

[20] R. Lindh, U. Ryde, M. Schütz, Theor. Chem. Acc. 97 (1997) 203.

[21] M. Svensson, S. Humbel, R.D.J. Froese, T. Matsubara, S. Sieber, K. Morokuma, J. Phys. Chem. 100 (1996) 19357.

[22] M.J. Frisch et al., Gaussian 98, Revision A.6, Gaussian Inc, Pittsburgh, PA, 1998.

[23] R.J. McMahon, R.J. Halter, R.L. Fimmen, R.J. Wilson, S.A. Peebles, R.L. Kuczkowski, J.F. Stanton, J. Am. Chem. Soc. 122 (2000) 939.

[24] R.A. Kumar, N. Ikemoto, D.J. Patel, J. Mol. Biol. 265 (1997) 173.

[25] R.A. Kumar, N. Ikemoto, D.J. Patel, J. Mol. Biol. 265 (1997) 187.

[26] J.P. Snyder, J. Am. Chem. Soc. 112 (1990) 5367.

[27] G.B. Jones, J.M. Wright, G. Plourde, A.D. Purohit, J.K. Wyatt, G. Hynd, F. Fouad, J. Am. Chem. Soc. 122 (2000) 9872.

[28] J.J. Devoss, J.J. Hangeland, C.A. Townsend, J. Am. Chem. Soc. 112 (1990) 4554.

[29] M. Schmittel, S. Kiau, Chem. Lett. (1995) 953.

[30] C.S. Kim, K.C. Russell, J. Org. Chem. 63 (1998) 8229.

[31] M.F. Semmelhack, J. Gallagher, Tetrahedron Lett. 34 (1993) 4121.

[32] P.G. Wenthold, R.R. Squires, W.C. Lineberger, J. Am. Chem. Soc. 120 (1998) 5279.

[33] W.R. Roth, H. Hopf, C. Horn, Chem. Ber. 127 (1994) 1765.

[34] W.R. Roth, H. Hopf, T. Wasser, H. Zimmerman, C. Werner, Liebigs Ann. (1996) 1691.

[35] S. Glasstone, K.J. Laidler, H. Eyring, in: The Theory of Rate Processes, McGraw-Hill, New York, 1941, p. 197. 